
\title{
$\begin{array}{ll}\text { Research Square } & \begin{array}{l}\text { Preprints are preliminary reports that have not undergone peer review. } \\ \text { They should not be considered conclusive, used to inform clinical practice, } \\ \text { or referenced by the media as validated information. }\end{array}\end{array}$
}

\section{Predicting Rifampicin Resistance Mutations in Bacterial RNA Polymerase Subunit Beta Based on Machine Learning Algorithms}

\author{
Qing Ning \\ Guangdong Key Laboratory of Environmental Pollution and Health, School of Environment, Jinan University \\ Dali Wang ( $\square$ wdali2018@jnu.edu.cn) \\ Guangdong Key Laboratory of Environmental Pollution and Health, School of Environment, Jinan University \\ Fei Cheng \\ Guangdong Key Laboratory of Environmental Pollution and Health, School of Environment, Jinan University \\ Yuheng Zhong \\ Guangdong Key Laboratory of Environmental Pollution and Health, School of Environment, Jinan University \\ Qi Ding \\ Guangdong Key Laboratory of Environmental Pollution and Health, School of Environment, Jinan University \\ Jing You \\ Guangdong Key Laboratory of Environmental Pollution and Health, School of Environment, Jinan University
}

\section{Research Article}

Keywords: Resistance mutation, machine learning, classifier, rifampicin, prediction

Posted Date: December 17th, 2020

DOI: https://doi.org/10.21203/rs.3.rs-127050/v1

License: (1) This work is licensed under a Creative Commons Attribution 4.0 International License. Read Full License

Version of Record: A version of this preprint was published at BMC Bioinformatics on April 22nd, 2021. See the published version at https://doi.org/10.1186/s12859-021-04137-0. 


\section{Abstract}

\section{Background}

Mutations in an enzyme target are one of the most common mechanisms whereby antibiotic resistance arises. Identification of the resistance mutations in bacteria is essential for understanding the structural basis of antibiotic resistance and design of new drugs. However, the traditionally used experimental approaches to identify resistance mutations were usually labor-intensive and costly.

\section{Results}

We present a machine learning (ML)-based classifier for predicting rifampicin (Rif) resistance mutations in bacterial RNA Polymerase subunit $\beta$ (RpoB). A total of 66 resistance mutations were gathered from the literature to form positive dataset, while 53 residue variations of RpoB among a series of naturally occurring species were obtained as negative database. The features of the mutated RpoB and their binding energies with Rif were calculated through computational methods, and used as the mutation attributes for modelling. Classifiers based on four $\mathrm{ML}$ algorithms, i.e. decision tree, $\mathrm{k}$ nearest neighbors, naïve Bayes and supporting vector machine, were developed, which showed accuracy ranging from 0.69 to 0.76 . A majority consensus approach was then used to obtain a new classifier based on the classifications of the four individual ML algorithms. The majority consensus classifier significantly improved the predictive performance, with accuracy, precision, recall and specificity of $0.83,0.84,0.86$ and 0.83 , respectively.

\section{Conclusion}

The majority consensus classifier provides an alternative methodology for rapid identification of resistance mutations in bacteria, which may help with early detection of antibiotic resistance and new drug discovery.

\section{Background}

Antibiotic resistance has become one of the greatest threats to public health all over the world. Pathogens with antibiotic resistance add difficulty to deal with infections and lead to increasing mortality. As stated by the United Nation in 2019 [1], at least 700 thousands of deaths are caused by infections of resistant pathogens every year, and this number will soar to 10 million annually by 2050 if no action is taken. Among the ever-growing resistant pathogens, Mycobacterium tuberculosis (MTB) is of particular concern because this species is a causative agent of tuberculosis, a highly-ranked death cause worldwide nowadays [1]. Rifampicin (Rif), an antibiotic of rifamycin class, has been extensively used to treat tuberculosis. However, there has been an increasing occurrence of Rif resistance in MTB, raising emerging health concerns [2, 3]. It was estimated that approximately 484,000 new cases of Rif-resistant tuberculosis and 214,000 Rif-resistant tuberculosis related deaths occurred in 2018 [4].

Antibiotic resistance in bacteria can originate from multiple sources, such as acquiring antibiotic resistance genes (ARGs) carried by mobile genetic elements (e.g. plasmids and transposons) [5], overexpression of multidrug efflux [6] and de novo resistance mutations in bacterial genomes [7]. For Rif, resistance is primarily caused by single point mutations in RNA polymerase (RNAP), an enzyme that is essential for RNA synthesis [8, 9]. Rif typically binds to the $\beta$ subunit of RNAP (RpoB) and blocks RNA synthesis, leading to the death of bacterial cells. Mutations in RpoB might cause changes of RpoB conformation and prevent Rif from binding to RpoB, resulting in loss of bactericidal activity of Rif. It should be noted that mutations occur randomly at any site of RpoB and do not always cause detrimental outcomes, instead, only those inducing resistance phenotypes (known as resistance mutations) are undesired and are more noteworthy. Currently, resistance mutations in bacteria are mostly identified through experimental approach, for example, to sequence and compare the relevant DNA segments in the mutants and the wild type strain, which are time- and labor-consuming. Since there are lots of probabilities for mutations in a given protein, it is of great significance to build predictive, other than experimental, approaches for quick screening of the resistance mutations.

Machine learning $(\mathrm{ML})$ is a branch of artificial intelligence (Al), which learns from massive amounts of data and reveals patterns and features in the data for predictions and decision making based on new data. Nowadays, ML algorithms have found applications in a variety of fields, such as speech recognition, traffic prediction and recommender systems [10-13]. In recent years, ML algorithms have been widely used in molecular biology and toxicology. For example, Murakami and Mizuguchi (2010) developed a naïve Bayes (NB) classifier for predicting the protein-protein interaction sites and Zhang et al. $(2017,2016)$ constructed NB classifiers for predicting drug-induced liver injury and mitochondrial toxicity in human. In those studies, ML algorithms served a powerful tool for solving classification problems. Classification is an important issue to understand the question whether mutations occurring in bacterial RpoB could lead to Rif resistance, and thus it would be useful for predicting the outcomes of these mutations, yet there have been rare such attempts in the literature. 
In this paper, we reported a novel ML-based method for predicting the Rif resistance mutations in bacterial RpoB. The positive resistance mutations were collected from the literature, while the negative mutations were obtained by gathering the residue variations in RpoB among different naturally occurring species. Four ML algorithms, i.e. decision tree (DT), k nearest neighbors (kNN), NB and supporting vector machine (SVM) were employed for modeling using 66 positive and 53 negative mutations. A majority consensus classifier was finally obtained based on the classifications of four individual ML algorithms, which showed satisfactory predictive performance. The ML-based classifier provides an alternative approach for quickly identifying Rif resistance mutations in bacteria.

\section{Results}

\section{Negative and positive mutations in RpoB of E. coli K12}

Mutations occur both spontaneously and under stresses during DNA replication and repair processes. Herein, mutations that confer bacterial resistance against antibiotics (i.e., antibiotic resistance mutations) are referred to as positive mutations, while those do not induce any changes in bacterial resistance phenotype are assigned as negative mutations. Resistance to Rif in bacteria is thought to be solely attributed to the single point mutations of $r p o B$ gene that encodes RpoB. Rif resistance mutations primarily occur within Rif resistancedetermining regions (RRDRs) of RpoB that are involved in the formation of the Rif-binding pocket (Fig. 1A). In the literature, the positive mutations were usually determined by sequencing the rpoB gene of the isolated Rif-resistant strain and comparing it with the wild type sequence. In the present research, 68 positive mutations at 25 amino acid positions were gathered from the literature. As shown in Fig. S1, a majority (17) of the sites were in RRDR- $\rrbracket$, while two in RRDR-N, five in RRDR- $₫$ and one in RRDR- $\rrbracket$. Among these residues, 516,526 and 531 in RRDR- $\mathbb{X}$ are the hotspots where resistance mutations occur most frequently [17].

Unlike the positive mutations, the negative mutations do not cause resistant phenotype. Moreover, negative mutations can be hardly recognized due to the difficulties in isolating the negative mutants and enriching them for sequencing. In the present research, we proposed that the differences of RRDRs in RpoB among different naturally occurring strains could be considered as negative mutations. This assumption was based on the facts that RRDRs in RpoB are highly conserved among different prokaryotes and play important roles in the combination of Rif and RpoB, and variations within RRDRs of RpoB from different naturally occurring strains may have no influence on the combination between RpoB and Rif. Therefore, the negative mutations in this work were obtained by finding out the different residues of RpoB among different strains. A total of 32021 RpoB sequences were downloaded from RefSeq database and 100 of them were randomly picked up to obtained a manageable number of the sequences. Figure 1B shows the sequence alignment of $E$. coli and other species (only 11 sequences were shown here), which suggested that the sequences had a very high identity in the range of RRDRs, with only a small minority of the sites displaying difference. The relationships between the number of the sampled sequences and the accumulative numbers of the different sites and the amino acid (AA) changes were described by the rarefaction curves. As shown in Fig. 1C, the number of the obtained sites and AA substitutions showed only slight changes when the number of the sequences approached to 100 , suggesting a satisfactory sampling depth. A total of 55 AA changes were finally gathered from the 100 sequences (Table 1), among which only two changes ( $\mathrm{H} 526 \mathrm{Q}$ and $\mathrm{H} 526 \mathrm{~N}$ ) had also appeared in the positive dataset. The two AA changes shared by the positive and negative datasets were excluded from both datasets before the subsequent calculation and analysis. Overall, there are 119 point-mutations in the final dataset, including 53 negative and 66 positive mutations.

Table 1

Negative and positive mutations in RNAP of E. coli

\begin{tabular}{|c|c|c|}
\hline Type & $\begin{array}{l}\text { Total } \\
\text { number }\end{array}$ & Amino acid changes \\
\hline Positive & 68 & $\begin{array}{l}\text { S512P V146F Q513K S522Y V146G L533P S512F I572F H526R Q148H H526L Q148K R529L Q513P S512Y } \\
\text { I572N G534C H526P Q148R I572T S508P G507D L533R S574Y I572S G534D Q148P G534S R687H T525R } \\
\text { R529S H526D P564L D516N G534V Q513R H526Y N518D S509R S531Y S531L G534A A532V T563P S512A } \\
\text { S522F D516Y L511Q L511R H526Q }{ }^{\mathrm{a}} \text { H526Na P564R E523V G570C D516G I572L S574F S531F I572M R529C } \\
\text { D516V A532E Q148L D516A Q513L R529H L511P L533H }\end{array}$ \\
\hline Negative & 55 & $\begin{array}{l}\text { N573T M685H I530M A689S S522A I569A Q686S S147N M515L N568S L575M S509G Q686M E523G S508A } \\
\text { I530L P567G S509N P567Q S531N I524L H526Q }{ }^{a} \text { H526Nª N573G P535I I530V P535E I145V L149M N573L } \\
\text { A683T G507S S508T N518T N518L S522G G507A I524M T525N N573S T563S P535Q G507T E523S Q510A } \\
\text { N518I S574T I524V E504S Q510R S147T A683S F506Y E504T S574N }\end{array}$ \\
\hline
\end{tabular}

Amino acid changes appeared in both negative and positive datasets were designated with a superscript "a" and shown in bold.

Evaluation of the mutated RpoB structures 
Twelve features (Table S1) were obtained by PremPS Server for each mutated RpoB. Among these features, $\Delta \Delta G$ is usually used for predicting the stability of the protein caused by mutations [18]. It is obtained by quantifying the change of unfolding Gibbs free energy $(\Delta G)$ of a protein after a single point mutation. According to the results, almost all (118) of the 119 mutants had greater $\Delta G$ s than the wild type RpoB (i.e., $\Delta \Delta G>0$ ), suggesting destabilizing effects of these point mutations (Fig. 2C). The only exception was A683S mutant with a $\Delta \Delta G$ value of $-0.61 \mathrm{kcal} \cdot \mathrm{mol}^{-1}$. Pearson's correlation coefficient of each pair of the features was shown in Fig. $2 \mathrm{~A}$, and the $p$ values were shown in Fig. 2B. It was revealed that, the stability of the mutated RpoB $(\Delta \Delta G)$ was significantly correlated $(p<0.01)$ to location, $P S S M, \triangle O M H, P \_L$, P_FWY, SASA_pro, SASA_sol, and the mutation type. As for the relationship between the mutation type and $\Delta \Delta \mathrm{G}$, it was shown in Fig. $2 \mathrm{C}$ that most of the $\Delta \Delta G$ values of the negative mutants fell into the range of $0-2.0 \mathrm{kcal} \cdot \mathrm{mol}^{-1}$, with a mean of $0.86 \mathrm{kcal} \cdot \mathrm{mol}^{-1}$, whereas $\Delta \Delta \mathrm{G}$ values of the positive mutants distributed in a larger range $\left(0-3.31 \mathrm{kcal} \bullet \mathrm{mol}^{-1}\right)$ and with a greater mean $\left(1.25 \mathrm{kcal}^{\circ} \mathrm{mol}^{-1}\right)$. Statistical analysis with an unpaired $t$ test (Mann Whitney test) suggested that the difference of the $\Delta \Delta G$ values between negative and positive mutations were statistically significant $(\mathrm{p}<0.01)$ (Fig. $2 \mathrm{C}$ ). This result implied that a mutant with a higher $\Delta \Delta \mathrm{G}$ value had a greater inclination to become resistant. With respect to the location, it was found that mutations at residues that were buried in the protein (i.e., in the core) tended to have greater $\Delta \Delta G$ values than those occurring on the surface of the protein (Fig. 2D). This suggested that changes of the buried residues may have greater destabilizing effects on the protein. The stability of the mutants also had a high correlation with P_FWY (Fig. 2E), which represented the fraction of aromatic residues buried in the protein core. This suggested that the aromatic residues buried in the protein core may have important functions in maintaining the stability of the protein.

\section{Interactions between mutated RpoB and Rif molecule}

The combination of Rif molecule with mutated and wild type RpoB were studied by molecular docking. The Rif pose obtained by docking simulation was similar to that obtained experimentally (i.e., Rif in the complex of 5UAC). As shown in Fig. 3A, the macrocyclic moieties of Rif in the two conformations presented a good overlap, while the 1-methylpiperazine tail of Rif in the two conformations showed a slight orientation change. Interactions between wild type RpoB and Rif molecule (in 5UAC complex) were shown in Fig. 3B. It can be seen that ARG529, SER531, ARG540 and PHE514 were key amino acids that involved in hydrogen bond interactions with Rif, while LEU511, LEU533 and PRO564 played important roles in the hydrophobic interactions. In addition, it was found that the macrocyclic moiety of Rif dominated in binding with RpoB, while the 1-methylpiperazine tail had no interaction with RpoB (Fig. 3B). In the docked complex (Fig. 3C), however, more interactions were found between RpoB and Rif, especially between RpoB and the 1-methylpiperazine tail of Rif. Specifically, GLU565 and ASN568 formed hydrogen bond interactions with the 1-methylpiperazine tail, and PRO567 formed a $\pi$-alkyl interaction with the piperazine ring. These interactions could well explain the orientation change of the 1-methylpiperazine tail in the docked complex, which was probably closer to the realistic situation. The structures of complexes of D516V and Rif, as well as S531L and Rif, have been determined experimentally in previous research [19]. Comparisons of the docked poses of Rif with the experimentally obtained conformations for D516V were shown in Fig. S2. As similar to the case of the wild type RpoB, the docked Rif in the D516V mutant also presented a slight change in the orientation of the 1-methylpiperazine tail, which was likely attributed to the interactions between the piperazine ring and the residues.

The distributions of the binding energies between Rif and mutated RpoB models were shown in Fig. 3D. The mean of the binding energies for negative mutants appeared lower than the positive mutants. Meanwhile, it was found that the binding energies between negative RpoB mutants and Rif was close to that between wild type RpoB and Rif $(-8.47 \mathrm{kcal} / \mathrm{mol})$, with only a few outliers (Fig. 3D). As for the positive mutants, however, the binding energies distributed in a wider range. This suggested that the positive mutants tended to present greater changes in the binding energies with Rif than the negative mutants.

\section{Comparison of classifiers using different machine learning algorithms}

Four classifiers with DT, kNN, NB and SVM algorithms were developed by using the PremPS-obtained attributes and the difference of the binding energies between wild type and mutated RpoB (denoted as $\triangle \mathrm{E}$ ). The overall confusion matrix for the classifiers and the detailed prediction results were shown in Table S2 and Table 2, respectively. The comparison of the prediction results of the classifiers were depicted in Fig. 4A. It was shown that kNN and NB classifiers had the greatest accuracy with a same score of 0.76 , followed by DT (0.71) and SVM (0.69). For kNN and NB classifiers, 91 of 119 mutations were predicted correctly, comparing to 84 and 82 correct predictions by DT and SVM, respectively. The precisions of the four classifiers, which represent the proportion of the correctly predicted positives, ranked as kNN $(0.79)>$ NB (0.75) > DT (0.73) > SVM (0.67). The recall (also known as sensitivity) and specificity, which represent the true positive rate and true negative rate, respectively, ranked differently for the four classifiers. Specifically, NB had the highest recall (0.88), followed by SVM (0.86), kNN (0.79) and DT (0.73), while kNN had the highest specificity (0.74), followed by DT (0.68), NB (0.62) and SVM (0.48). In general, the recall was higher than the specificity for all of the classifiers, suggesting a greater ability of the classifiers in correctly identifying the positive mutations. For example, 58 of 66 positive mutations were correctly classified by NB model, whereas only 33 of 53 negative mutations were correctly identified by this model. It should be noted that, the predictive models developed herein are intended to identify the potential 
resistance mutations (i.e., positive mutations), thus it is more important that the positive mutations are predicted correctly. In view of this, the recalls of the classifiers are more important than the specificity. F-measure, which conveys the balance between recall and precision, was also calculated. As listed in Table 2, NB had the highest F-measure score (0.81), followed by kNN (0.79), SVM (0.75) and DT (0.72). Furthermore, the receiver operating characteristic (ROC) curves of the classifiers (Fig. 4B), which demonstrated the connection between recall and specificity, were obtained by plotting the recall versus "1-specificity" across all possible thresholds for the test. A ROC curve that is closer to the left-hand and top borders of the ROC space points to a high accuracy of the classifier, and the area under the ROC curve (AUC) was used to represent the measure of separability of the classifiers. According to the results (Table 2), kNN, NB and SVM had similar AUC scores $(0.81,0.80$ and 0.80 , respectively), suggesting close separation capacity of the three classifiers, which was greater than DT whose AUC score was 0.74 .

Table 2

Prediction results of the four developed classifiers. DT: decision tree; kNN: k nearest neighbors; NB: naïve bayes; SVM: supporting vector machine; MC: majority consensus. AUC: area under the curve. Data are presented as mean \pm standard deviation.

\begin{tabular}{|lllllll|}
\hline Classifier & Accuracy & AUC & F-measure & Precision & Recall & Specificity \\
\hline DT & $0.71(0.13)$ & $0.74(0.11)$ & $0.72(0.14)$ & $0.73(0.10)$ & $0.73(0.19)$ & $0.68(0.07)$ \\
\hline kNN & $0.76(0.10)$ & $0.81(0.08)$ & $0.79(0.10)$ & $0.79(0.12)$ & $0.79(0.11)$ & $0.74(0.14)$ \\
NB & $0.76(0.05)$ & $0.80(0.10)$ & $0.81(0.03)$ & $0.75(0.07)$ & $0.88(0.05)$ & $0.62(0.14)$ \\
SVM & $0.69(0.13)$ & $0.80(0.08)$ & $0.75(0.11)$ & $0.67(0.10)$ & $0.86(0.13)$ & $0.48(0.14)$ \\
MC & $0.83(0.09)$ & $0.86(0.08)$ & $0.85(0.08)$ & $0.86(0.06)$ & $0.84(0.12)$ & $0.83(0.08)$ \\
\hline
\end{tabular}

\section{Evaluation of the majority consensus prediction}

The majority consensus prediction was based on the classification results of the four individual ML algorithms. The prediction results and ROC curves for the majority consensus were shown in Table 2 and Fig. 4B, respectively. In general, the majority consensus showed better predictive performance than the four individual $\mathrm{ML}$ algorithms, with higher accuracy, AUC, F-measure, precision and specificity scores (Fig. 4A). The accuracy of the majority consensus was 0.83 , with 99 of 119 mutations being correctly predicted. In particular, the majority consensus prediction had a reasonably balanced recall and specificity, which were 0.84 and 0.83 , respectively. That is, 55 positive mutations were identified from a total of 66 data, and 44 negative mutations were identified from 53 data. The precision, which represents the correct proportion of positive identifications, came out to be higher for the majority consensus with a score of 0.86 , and the F-measure, which balances the concerns of recall and precision was also higher for the majority consensus (0.85) than the individual algorithms.

\section{Application of the classifiers}

In theory, a total of $1197(63 \times 19)$ single point mutations can occur within the RRDRs of RpoB (19 possibilities for each site). All of the possible RpoB mutants were built through PremPS server and were investigated for their interactions with Rif molecule by LeDock. The PremPS-obtained features and the $\triangle \mathrm{E}$ for the RpoB mutants were provided in the Table S3. Afterwards, the resistance types of the mutated RpoB were predicted by using four individual ML algorithms, i.e. DT, kNN, NB and SVM. A total of $685,684,917$ and 1100 positive mutations were identified by the four algorithms, respectively. Then the classifications of the four ML algorithms were combined based on the majority consensus to obtain the final classification. The majority consensus identified 820 positive mutations, and the final classifications were depicted as a heatmap in Fig. 5. It was shown that the resistance mutations could occur at a majority of the sites within RRDRs region, with exceptions of GLU504, GLN510, PHE514, GLU565, PR0567, ILE569, LEU575, ASN684, where no resistance mutations were predicted. However, it should be noted that the predicted resistance mutations shown in Fig. 5 do not necessarily occur in real conditions, they only represent that the mutations have a high probability to confer resistance phenotype if they occur.

\section{Discussion}

Antibiotic resistance is a major threat to public health and has aroused great concerns. One of the mechanisms for antibiotic resistance is the mutations occurring in an enzyme target, causing structural modifications of the active sites that no longer allow the combination of the antibiotics [20]. Resistance originating in this way has been observed for many antibiotics. For example, mutations of $p b p$, a gene encoding penicillin-binding proteins, conferred bacteria with $\beta$-lactams resistance [21], and mutations of $r$ rnA and $r r n B$, genes that encode $16 S$ rRNA, conferred tetracycline resistance [22]. In the case of Rif, resistance usually arises as a result of single point mutations in bacterial RNAP, an enzyme that accommodates Rif molecule at the $\beta$ subunit. Identification of the resistance mutations is essential for understanding the structural basis of the antibiotic resistance, and provides useful information for prevention of the resistance emergence and new drug discovery. In previous studies, the identification of the resistance mutations was usually achieved through labor-intensive and costly

Page 5/17 
experiment, for example to isolate the resistance mutants and sequence the relevant gene segments followed by aligning the sequences with those of the sensitive strains [23,24]. The experimental approach is thus incapable of rapid identification, and moreover, the identification with experimental approach usually lags behind the emergence of the resistance. Therefore, prediction of the resistance mutations based on known information is highly desired.

In previous studies, there have been some attempts that focused on the prediction of antibiotic resistance mutations. For example, Frey et al. (2010) reported a computational approach for predicting resistance mutations in dihydrofolate reductase of bacteria. In this approach, a protein design algorithm was employed for generating positive and negative mutations and the prediction was based on the analysis of the affinity between the mutants and the inhibitor. Comparing to this approach, the predictive algorithm developed in the present work takes into account not only the affinity but also a series of features of the mutants. Moreover, the predictive algorithms in the present work were trained using a collection of both negative and positive mutations, and is thus more reliable and explicable.

There haven't been any attempts in the literature to use ML algorithms for predicting resistance mutations, until recently Jamal et al. (2020) reported a work that used Al and ML algorithms to predict resistant and susceptible mutations in MTB. However, the method for developing predictive models in Jamal et al. (2020) was debatable. When preparing the database for modeling, the authors labeled mutations with positive $\Delta \Delta G$ values as susceptible mutations, otherwise as resistant mutations. The problem was that $\Delta \Delta G$ was an indicator of the stability of a mutated protein, and it was improper to use this parameter alone for classifying the mutations, although this parameter might have some relationship with the mutation type. Moreover, in the following steps, the authors used this parameter again as an attribute of the mutations for training the classification models, which was also improper. Unlike this work, the data used for developing the predictive models in the present research came from two sources, the resistance mutations that have been validated experimentally were obtained from the literature and used as the positive dataset, while the different residues of RNAPs from a series of naturally occurring species were deemed as neutral mutations and used for the negative dataset. In addition, we developed a majority consensus algorithm based on the predictions of four individual ML algorithms, which significantly improved the predictive performance. However, the classifier developed in this work still has some limitations, for example, it can only apply to predicting Rif resistance mutations and the classifications need further validation with experiments. Nonetheless, the present work provides an inspiration and an alternative methodology for rapid identification of resistance mutations in bacteria, which may be helpful for early detection of resistance and new drug discovery.

\section{Conclusion}

A majority consensus classifier was developed for predicting Rif resistance mutations in bacterial RNAP based on four ML algorithms (i.e. $\mathrm{DT}, \mathrm{kNN}, \mathrm{NB}$ and SVM), using 66 positive resistance mutations and 53 negative mutations as the dataset. The features of the mutated RpoB and their combination with Rif molecule were studied by computational approaches and used for developing the predictive models. Analysis of the features showed that the mutation type was significantly related to $\triangle \Delta G$, PSSM and P_FWY. Estimates of the predictive models showed that the four individual algorithms presented varying predictive performance with accuracy ranging from 0.69 to 0.76 , while the majority consensus classifier gave better predictions with a higher accuracy of 0.83 , and higher AUC (0.86), higher precision (0.86) and specificity (0.83) as well. Using this majority consensus, the possible mutations within RRDRs of RNAP were classified, which showed that a majority of the mutations were identified as resistance-conferring mutations. However, it should be noted that further experiment is needed for validation of the classifications.

\section{Methods}

\section{Mutation dataset preparation}

Mutations in RpoB that confer bacteria with Rif resistance are assigned as positive, otherwise as negative. The positive mutations in RpoB of $E$. coli have been well documented in the literature $[19,27]$ and were gathered to obtain the positive dataset. As shown in Table 1, the positive dataset included 68 amino acid (AA) substitutions at 25 sites in total. All of the 68 AA changes were within the RRDRs (Table S4) [19], which were considered highly conserved among prokaryotes. It should be noted that the AA changes gathered in the positive dataset were not necessarily exhaustive, because some changes that confer Rif resistance may have not been observed or reported yet. Despite of the high conservation of the RRDRs, differences in the residues of the RRDRs may still exist among naturally occurring species. These differences are unlikely to affect the susceptibility of the species against Rif and can thus be deemed as negative mutations. Therefore, the negative dataset used for the machine learning was obtained through the following procedures. First, the sequences of RpoB from different bacteria were downloaded from NCBI reference sequence (RefSeq) database, filtered with "bacteria" in species and "1000-1400" in sequence length. A total of 32021 sequences were obtained in this way, and 100 of them were picked up randomly and subjected to alignment with the RpoB sequence of $E$. coli. Afterwards, the different residues within RRDRs between $E$. coli and other species were 
gathered to obtained the negative dataset. The sequence alignment was conducted on Discovery Studio (DS) by using Align Sequences protocol. The negative database was shown in Table 1.

\section{Construction of the RpoB mutants}

The wildtype RpoB in E. coli K12 was obtained from Protein Data Bank (ID: 5UAC, Chain C). Mutated RpoB with single point mutations were constructed through PremPS Server (https://lilab.jysw.suda.edu.cn/research/PremPS/), using the wildtype RpoB (5UAC) as a template. PremPS Server also gives a set of parameters that characterize the mutated proteins, such as the unfolding Gibbs free energy changes $(\Delta \Delta G)$, differences of hydrophobicity scale between mutated and wildtype RpoB $(\triangle \mathrm{OMH})$, and the solvent-accessible surface area (SASA_pro) [28]. Interpretations of these parameters are listed in Table S1.

\section{Molecular docking}

The interactions between Rif molecule and mutated and wild type RNAP were investigated by LeDock, a molecular docking program [29]. Prior to the docking, the wild type RpoB and the mutated models were prepared by LePro, which added missing hydrogen to the proteins and remove redundant structures. The binding pocket of the protein was set manually as a cube box at the same location for both mutated and wild type RpoB, with coordinates of $x=105-123, y=20-38$, and $z=-17-1$. The number of the binding poses was set as 20 , which means the process will generate 20 random docking poses. The poses with the highest scores were chosen to represent the optimal binding poses of Rif with the mutated models.

\section{Machine learning algorithms}

Four supervised machine learning (ML) algorithms were employed for developing predictive models in the present research, i.e. Naïve Bayes (NB), k nearest neighbor (kNN), support vector machine (SVM) and decision tree (DT). Naive Bayes (NB) classifier is a supervised learning algorithm based on Bayes' theorem with a "naive" assumption that all attributes are independent given the value of the class variable [30, 31]. kNN classifier is based on the Euclidean distance between the target sample and the training samples, where $k$ denotes the number of the nearest neighbors that are used for classifying the target sample [32]. SVM is a statistical learning method that uses a hyperplane to optimally separate data into negative and positive categories [33]. DT predicts the category of a sample by using a tree-like flowchart, where the nodes represent the test on an attribute and the branches denote the outcome of the test [34]. All of the four ML algorithm are commonly used for solving classification problems. In the present research, the predictive models using the four ML algorithms were developed on KNIME platform with "Naïve Bayes Learner", "SVM Learner”, “K Nearest Neighbor” and "Decision Tree Learner” nodes, respectively. Cross validation with $\mathrm{K}$-fold = 4 was performed by using a combination of "X-partitioner" and "X-aggregator" nodes. Stratified sampling was used for partitioning the total data in X-partitioner node. Receiver Operating Characteristic (ROC) curve was generated using a "ROC curve" node. Mean and standard deviation of the precision (P), recall (R), specificity (SP) accuracy (AC) and F-measure were calculated and gathered for evaluation of the NB classifier. The workflow used for developing the predictive models was depicted in Fig. S3. The functions used for deriving these parameters were as follows.

$$
\begin{gathered}
P=\frac{T P}{T P+F P} \\
R=\frac{T P}{T P+F N} \\
S P=\frac{T N}{T N+F P} \\
A C=\frac{T P+T N}{T P+F P+T N+F N} \\
F \text {-measure }=\frac{2 \times P \times R}{P+R}
\end{gathered}
$$

Where TP, FP, TN and FN denote true positive, false positive, true negative and false negative respectively. 


\section{Majority Consensus}

A majority consensus approach was used to obtain a new classifier, which combined the classification results of the four individual ML algorithms. In this approach, a positive prediction was assigned to a mutation when 3 or 4 of the individual algorithms gave positive predications, otherwise a negative prediction was assigned to this mutation.

\section{Abbreviations}

AA: Amino acid; Al: Artificial intelligence; ARGs: Antibiotic resistance genes; AUC: Area under the curve; DNA: Deoxyribonucleic acid; DT: Decision tree; E. coli: Escherichia coli; kNN: k nearest neighbors; MC: Majority consensus; ML: Machine learning; MTB: Mycobacterium tuberculosis; NB: naïve Bayes; NCBI: National Center for Biotechnology Information; Rif: Rifampicin; RNA: Ribonucleic acid; RNAP: RNA polymerase; ROC: Receiver operating characteristic; RpoB: RNA Polymerase subunit $\beta$; RRDRs: Rif resistance-determining regions; SVM: Supporting vector machine; $\Delta \Delta \mathrm{G}$ : unfolding Gibbs free energy changes.

\section{Declarations}

\section{Ethics approval and consent to participate}

Not applicable.

\section{Consent for publication}

Not applicable.

\section{Availability of data and materials}

The datasets used during the current study are available from the corresponding author on reasonable request.

\section{Competing interests}

The authors declare that they have no competing interests

\section{Funding}

This work was financially supported by the National Natural Science Foundation of China (21806055), the Guangdong Provincial Department of Science and Technology (2019A1515011583) and the National Natural Science Foundation of China (U1901220).

\section{Authors' contributions}

QN drafted the manuscript. DW, FC and JY revised the manuscript. DW, FC and JY conceptualized the methods. DW designed the experiments, while QN, YZ, QD performed the experiments. DW and JY supervised the design and analysis of methods. All authors provided critical feedback on the manuscript, read and approved the final manuscript.

\section{Acknowledgements}

PremPS was employed for calculating the features of the mutated RpoB. This program is developed by Minghui Li Research Group at Soochow University and is freely available online. We thank Dr. Li for his contributions.

\section{References}


1. Interagency Coordination Group on Antimicrobial Resistance. No time to wait: securing the future from drug-resistant infections. Report to the secretary-general of the United Nations. 2019. https://www.who.int/antimicrobial-resistance/interagency-coordinationgroup/IACG_final_report_EN.pdf?ua=1. Accessed 10 Dec 2020.

2. Gillespie SH. Evolution of drug resistance in Mycobacterium tuberculosis: clinical and molecular perspective. Antimicrob Agents Chemother. 2002;46:267-74.

3. Taniguchi H, Aramaki H, Nikaido Y, Mizuguchi Y, Nakamura M, Koga T, et al. Rifampicin resistance and mutation of the rpoB gene in Mycobacterium tuberculosis. FEMS Microbiol Lett. 1996;144:103-8.

4. MacNeil A. Global Epidemiology of Tuberculosis and Progress Toward Meeting Global Targets-Worldwide, 2018. MMWR Morb Mortal Wkly Rep. 2020;69.

5. Partridge SR, Kwong SM, Firth N, Jensen SO. Mobile genetic elements associated with antimicrobial resistance. Clin Microbiol Rev. 2018;31.

6. Alcalde-Rico M, Hernando-Amado S, Blanco P, Martínez JL. Multidrug efflux pumps at the crossroad between antibiotic resistance and bacterial virulence. Front Microbiol. 2016;7:1483.

7. Zhang Q, Lambert G, Liao D, Kim H, Robin K, Tung CK, et al. Acceleration of emergence of bacterial antibiotic resistance in connected microenvironments. Science (80-). 2011;333:1764-7.

8. Campbell EA, Korzheva N, Mustaev A, Murakami K, Nair S, Goldfarb A, et al. Structural mechanism for rifampicin inhibition of bacterial RNA polymerase. Cell. 2001;104:901-12.

9. Goldstein BP. Resistance to rifampicin: a review. J Antibiot (Tokyo). 2014;67:625-30.

10. Rzeszótko J, Nguyen SH. Machine learning for traffic prediction. Fundam Informaticae. 2012;119:407-20.

11. Padmanabhan J, Johnson Premkumar MJ. Machine learning in automatic speech recognition: A survey. IETE Tech Rev. 2015;32:24051.

12. Deng L, Li X. Machine learning paradigms for speech recognition: An overview. IEEE Trans Audio Speech Lang Processing. 2013;21:1060-89.

13. Portugal I, Alencar P, Cowan D. The use of machine learning algorithms in recommender systems: A systematic review. Expert Syst Appl. 2018;97:205-27.

14. Murakami Y, Mizuguchi K. Applying the Naïve Bayes classifier with kernel density estimation to the prediction of protein-protein interaction sites. Bioinformatics. 2010;26:1841-8.

15. Zhang H, Yu P, Ren J-X, Li X-B, Wang H-L, Ding L, et al. Development of novel prediction model for drug-induced mitochondrial toxicity by using naïve Bayes classifier method. Food Chem Toxicol. 2017;110:122-9.

16. Zhang H, Ding L, Zou Y, Hu S-Q, Huang H-G, Kong W-B, et al. Predicting drug-induced liver injury in human with Naïve Bayes classifier approach. J Comput Aided Mol Des. 2016;30:889-98.

17. Lahiri N, Shah RR, Layre E, Young D, Ford C, Murray MB, et al. Rifampin resistance mutations are associated with broad chemical remodeling of Mycobacterium tuberculosis. J Biol Chem. 2016;291:14248-56.

18. Zhang Z, Wang L, Gao Y, Zhang J, Zhenirovskyy M, Alexov E. Predicting folding free energy changes upon single point mutations. Bioinformatics. 2012;28:664-71.

19. Molodtsov V, Scharf NT, Stefan MA, Garcia GA, Murakami KS. Structural basis for rifamycin resistance of bacterial RNA polymerase by the three most clinically important RpoB mutations found in Mycobacterium tuberculosis. Mol Microbiol. 2017;103:1034-45.

20. Woodford N, Ellington MJ. The emergence of antibiotic resistance by mutation. Clin Microbiol Infect. 2007;13:5-18.

21. Mascher T, Heintz M, Zähner D, Merai M, Hakenbeck R. The CiaRH System of Streptococcus pneumoniae Prevents Lysis during Stress Induced by Treatment with Cell Wall Inhibitors and by Mutations in pbp2x Involved in $\beta$-Lactam Resistance. J Bacteriol. 2006;188:1959.

22. Wu JY, Kim JJ, Reddy R, Wang WM, Graham DY, Kwon DH. Tetracycline-resistant clinical Helicobacter pylori isolates with and without mutations in 16S rRNA-encoding genes. Antimicrob Agents Chemother. 2005;49:578-83.

http://gateway.isiknowledge.com/gateway/Gateway.cgi?

GWVersion=2\&SrcAuth=AegeanSoftware\&SrcApp=NoteExpress\&DestLinkType=FullRecord\&DestApp=WOS\&KeyUT=000226709900016.

23. Herrera L, Jiménez S, Valverde A, García-Aranda MA, Sáez-Nieto JA. Molecular analysis of rifampicin-resistant Mycobacterium tuberculosis isolated in Spain (1996-2001). Description of new mutations in the rpoB gene and review of the literature. Int $\mathrm{J}$ Antimicrob Agents. 2003;21:403-8.

24. Lv L, Jiang T, Zhang S, Yu X. Exposure to Mutagenic Disinfection Byproducts Leads to Increase of Antibiotic Resistance in Pseudomonas aeruginosa. Environ Sci Technol. 2014;48:8188-95. 
25. Frey KM, Georgiev I, Donald BR, Anderson AC. Predicting resistance mutations using protein design algorithms. Proc Natl Acad Sci. 2010;107:13707-12.

26. Jamal S, Khubaib M, Gangwar R, Grover S, Grover A, Hasnain SE. Artificial Intelligence and Machine learning based prediction of resistant and susceptible mutations in Mycobacterium tuberculosis. Sci Rep. 2020;10.

27. Garibyan L, Huang T, Kim M, Wolff E, Nguyen A, Nguyen T, et al. Use of the rpoB gene to determine the specificity of base substitution mutations on the Escherichia coli chromosome. DNA Repair (Amst). 2003;2:593-608.

28. Chen Y, Lu H, Zhang N, Zhu Z, Wang S, Li M. PremPS: Predicting the Effects of Single Mutations on Protein Stability. bioRxiv. 2020.

29. Wang Z, Sun H, Yao X, Li D, Xu L, Li Y, et al. Comprehensive evaluation of ten docking programs on a diverse set of protein-ligand complexes: the prediction accuracy of sampling power and scoring power. Phys Chem Chem Phys. 2016;18:12964-75.

30. Berger JO. Statistical decision theory and Bayesian analysis. Springer Science \& Business Media; 2013.

31. Box GEP, Tiao GC. Bayesian inference in statistical analysis. John Wiley \& Sons; 2011.

32. Kataria A, Singh MD. A review of data classification using k-nearest neighbour algorithm. Int J Emerg Technol Adv Eng. 2013;3:354-60.

33. Noble WS. What is a support vector machine? Nat Biotechnol. 2006;24:1565-7.

34. Song Y-Y, Ying LU. Decision tree methods: applications for classification and prediction. Shanghai Arch psychiatry. 2015;27:130.

\section{Figures}

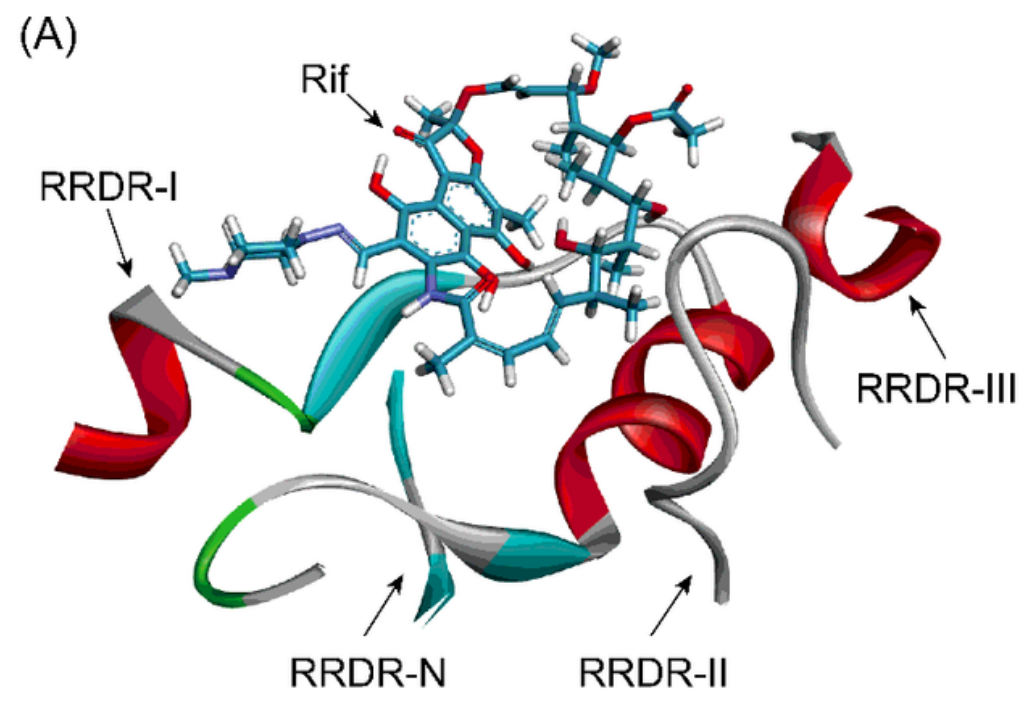

(B)

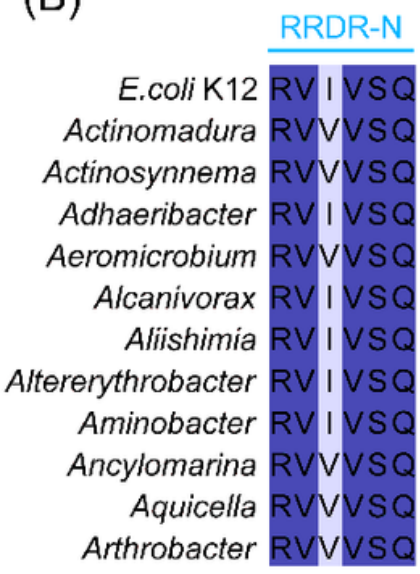

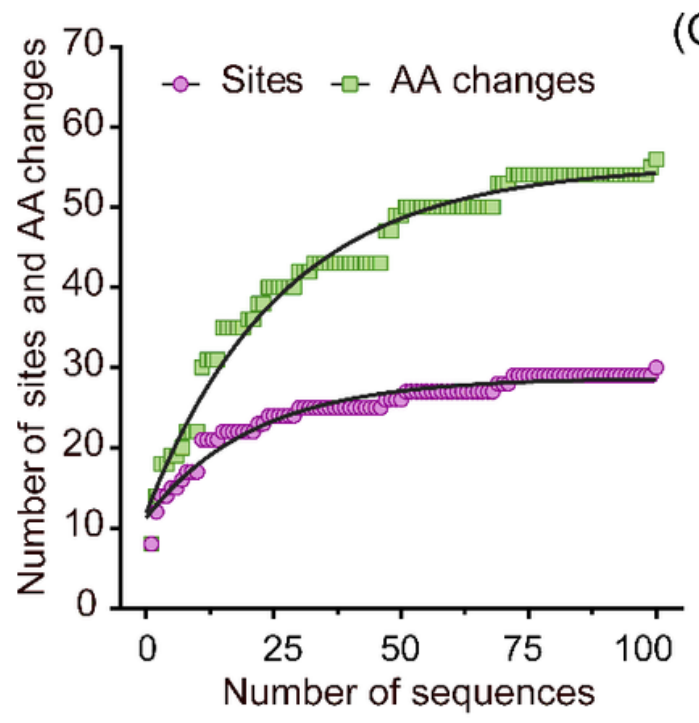

(C) 
(A) A side view of the structure of the rifampin (Rif) binding pocket formed by the Rif resistance-determining regions (RRDRs) of RpoB. (B) Sequence alignment spanning RRDRs of E. coli and other 11 species. (C) Rarefaction curves that describe the relationship between the number of the sequences and the accumulative number of the obtained different sites and amino acid (AA) changes.
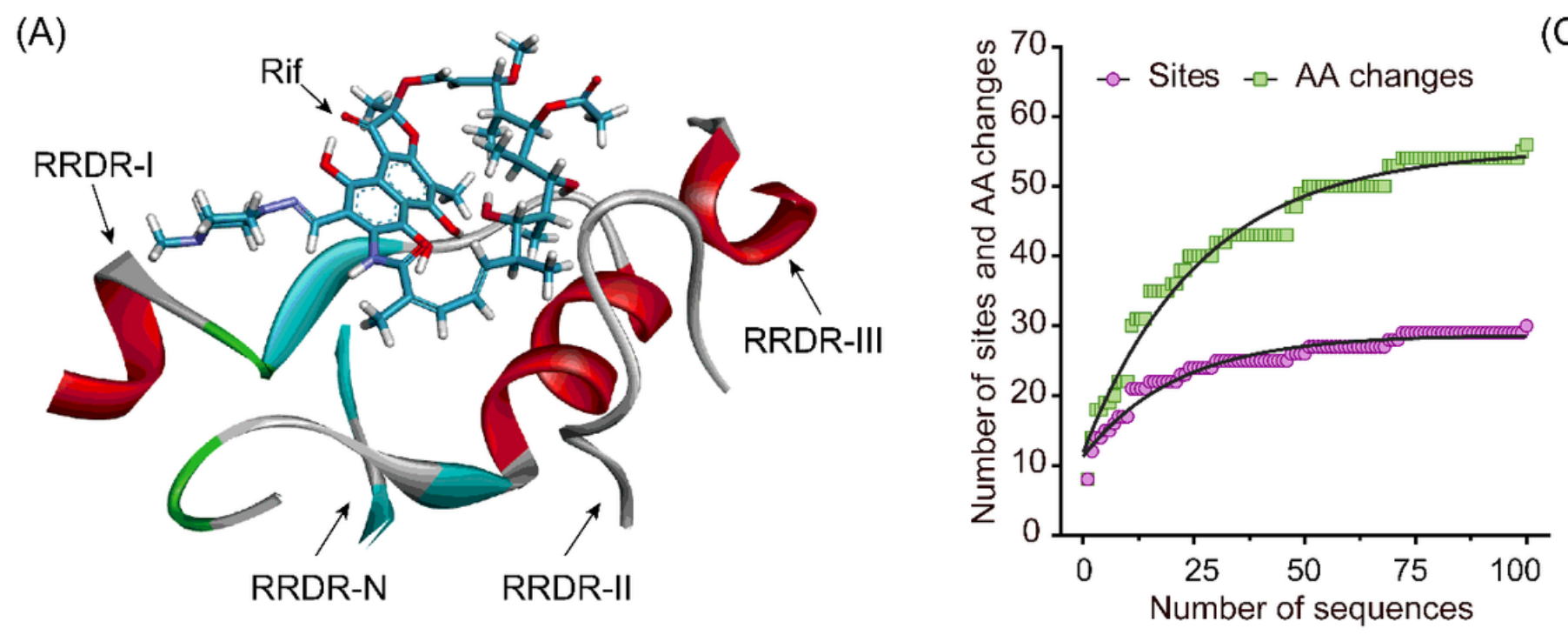

(B)
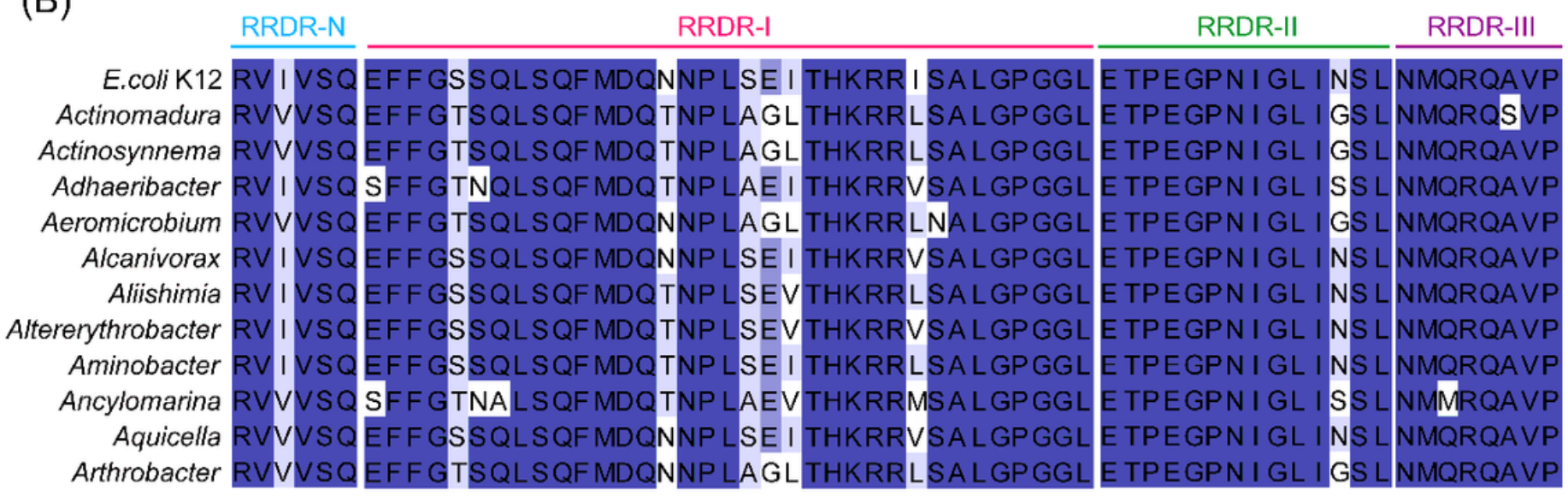

Figure 1

(A) A side view of the structure of the rifampin (Rif) binding pocket formed by the Rif resistance-determining regions (RRDRs) of RpoB. (B) Sequence alignment spanning RRDRs of E. coli and other 11 species. (C) Rarefaction curves that describe the relationship between the number of the sequences and the accumulative number of the obtained different sites and amino acid (AA) changes. 

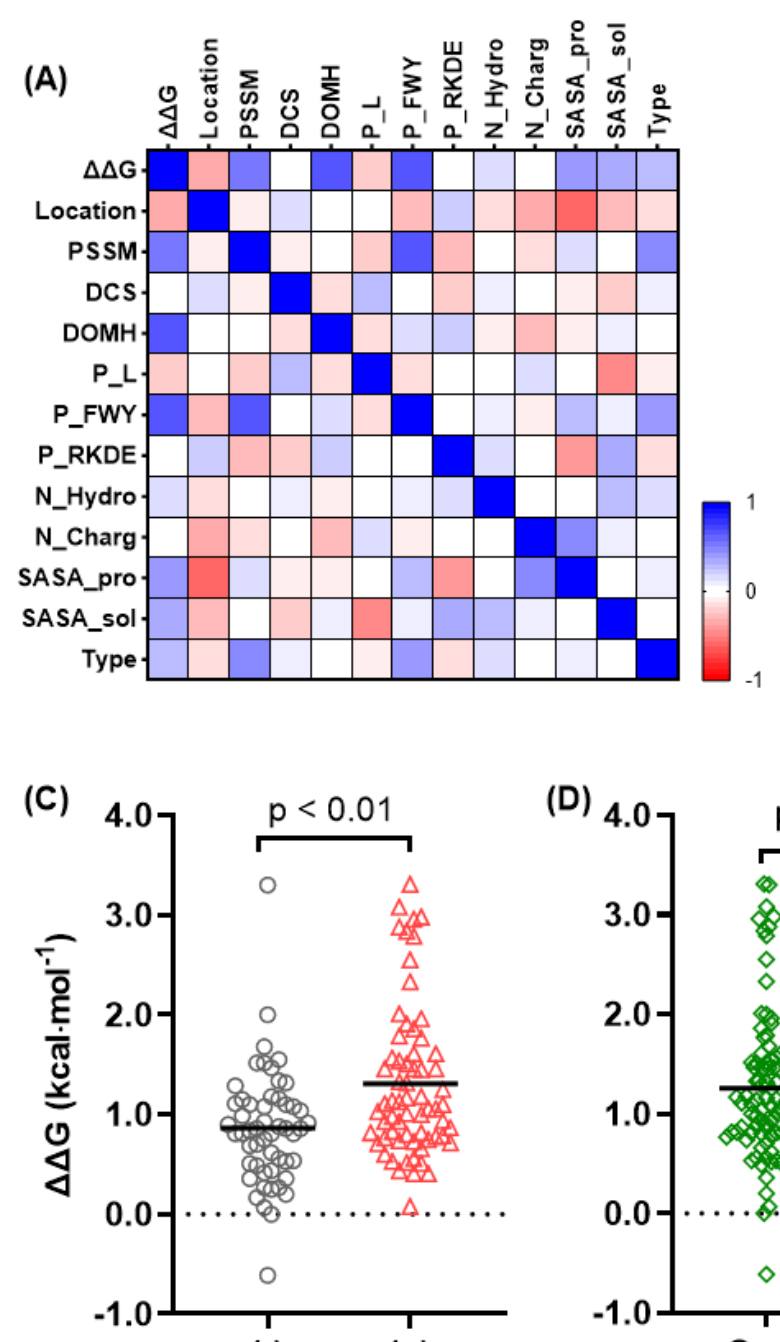

$(-) \quad(+)$

Mutation type
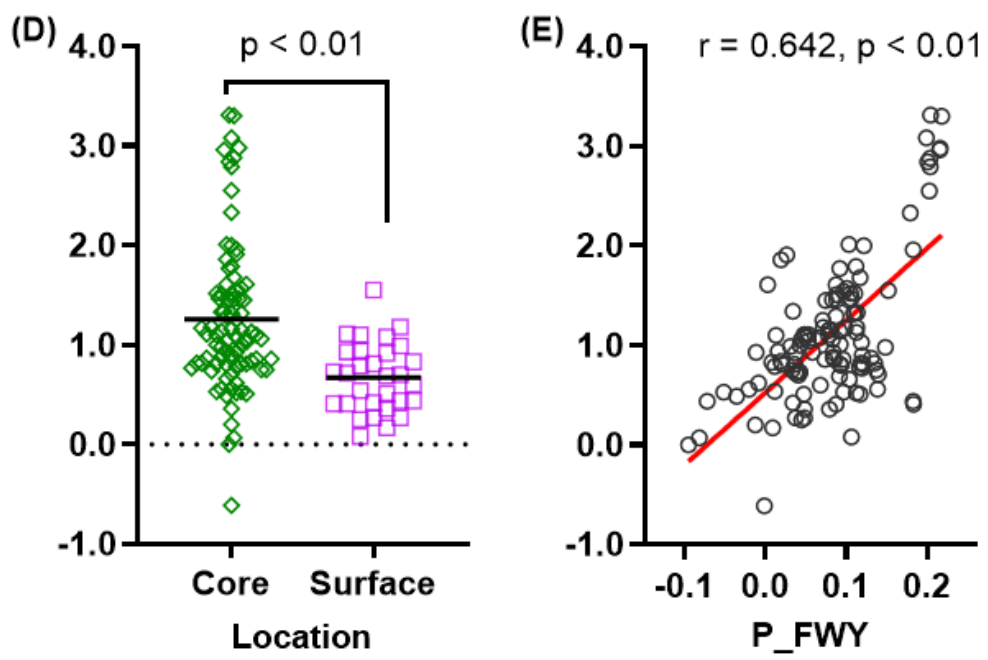

Figure 2

(A) Correlation matrix for the features of the mutated RpoB. The correlation of each pair of the features was quantified by a Pearson's correlation coefficient, which ranges from -1 to 1 . (B) p values for the correlation matrix. (C) Correlation of the stability of the mutants $(\Delta \Delta G)$ with the mutation type. The signs "-" and "+" denote negative and positive mutations, respectively. (D) Correlation of $\Delta \Delta G$ with location. (E) Correlation of $\Delta \Delta G$ with P_FWY (fraction of aromatic residues buried in the protein core). 

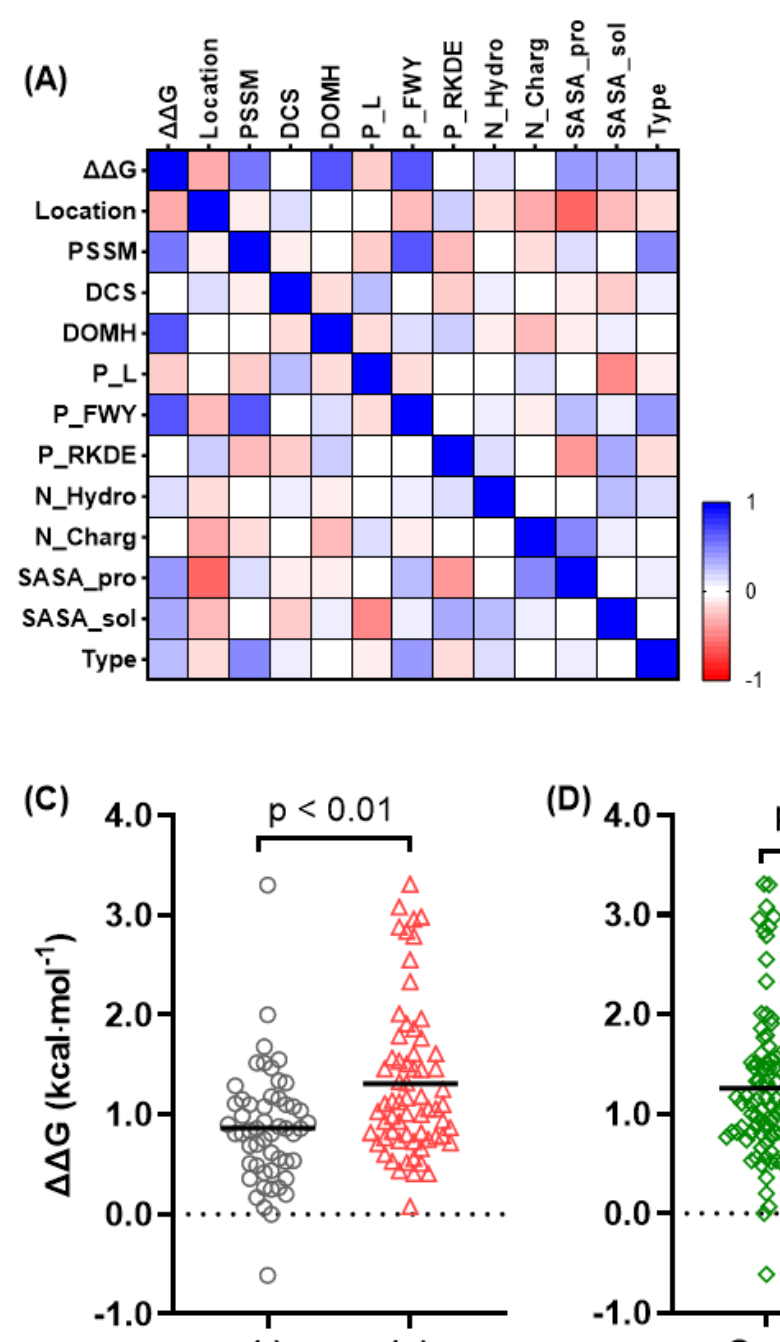

$(-) \quad(+)$

Mutation type
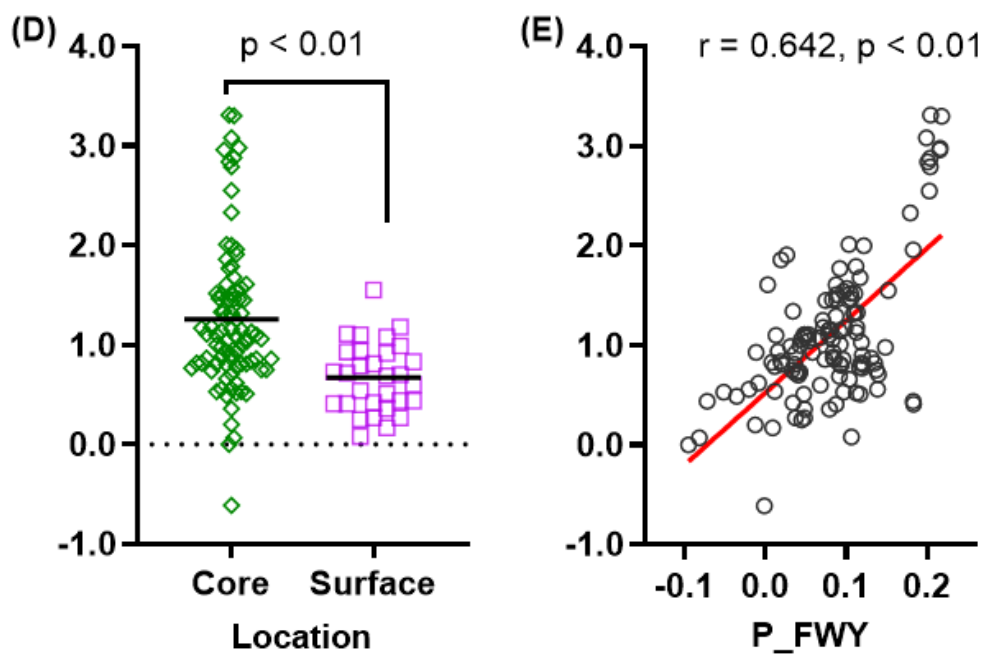

Figure 2

(A) Correlation matrix for the features of the mutated RpoB. The correlation of each pair of the features was quantified by a Pearson's correlation coefficient, which ranges from -1 to 1 . (B) p values for the correlation matrix. (C) Correlation of the stability of the mutants $(\Delta \Delta G)$ with the mutation type. The signs "-" and "+" denote negative and positive mutations, respectively. (D) Correlation of $\Delta \Delta G$ with location. (E) Correlation of $\Delta \Delta G$ with P_FWY (fraction of aromatic residues buried in the protein core). 
(A)

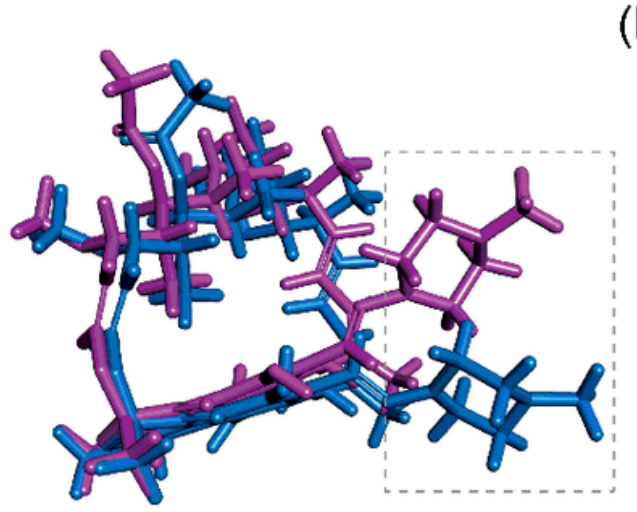

(D)

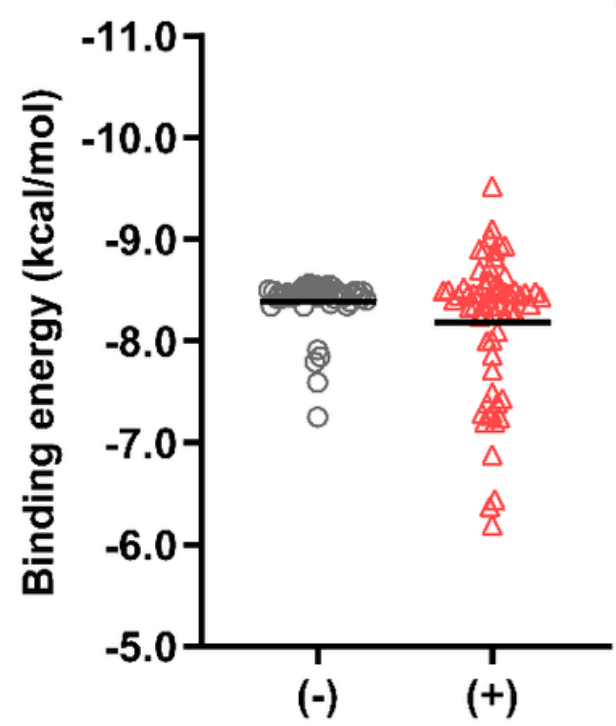

(B)

(C)
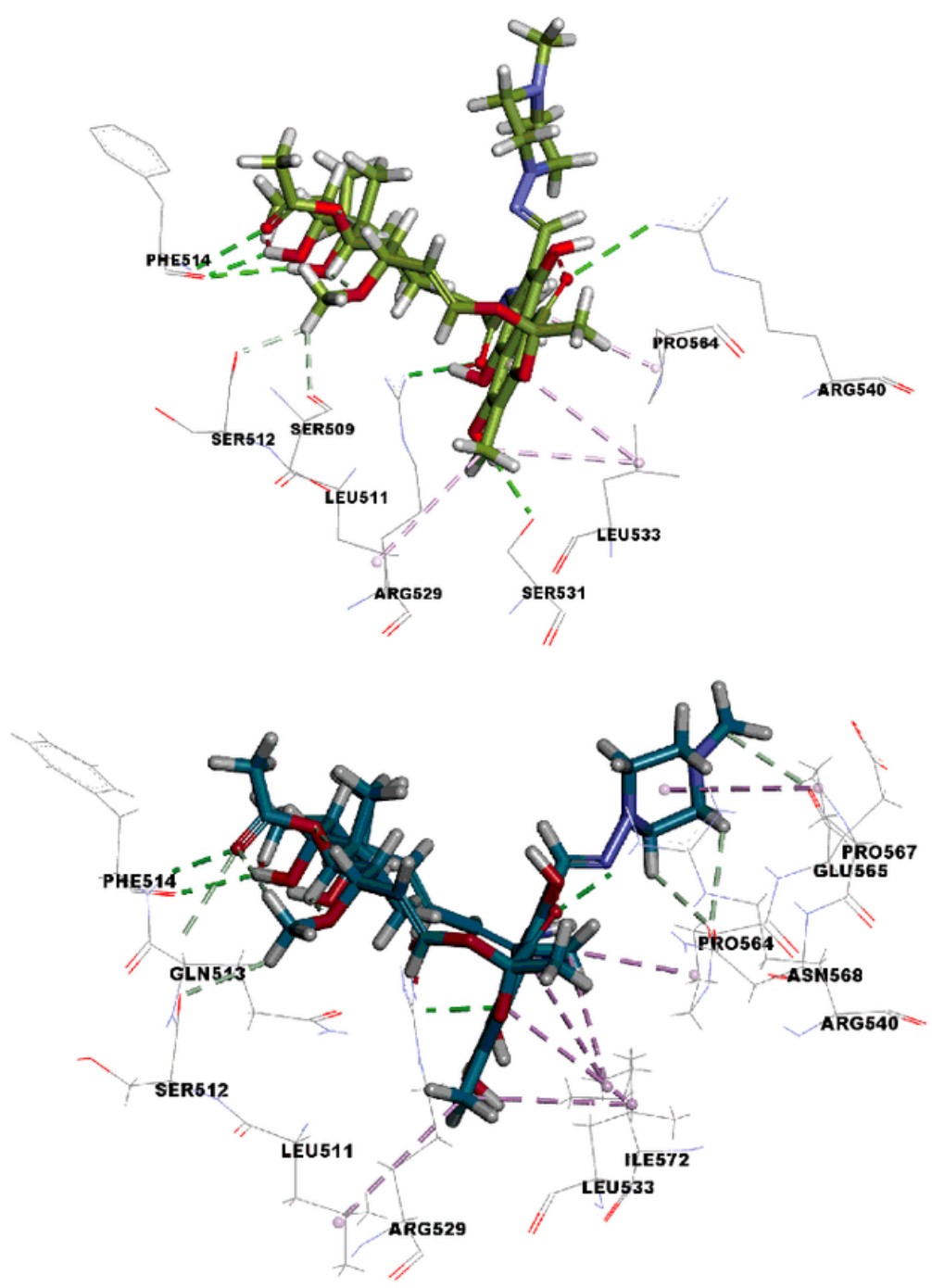

Mutation type

Figure 3

(A) Comparison of the Rifampin (Rif) poses obtained by molecular docking in the present research (purple) and obtained experimentally in the literature (blue). The structure in the dashed box is the 1-methylpiperazine tail of Rif. (B) Interactions between wild type RpoB and Rif molecule in the RpoB-Rif complex obtained from the Protein Database (5UAC). (C) Interactions between wild type RpoB and Rif molecule in RpoB-Rif complex obtained by molecular docking. (D) Distributions of the binding energies of Rif with RpoB mutants. The straight lines indicate the mean values. 
(A)

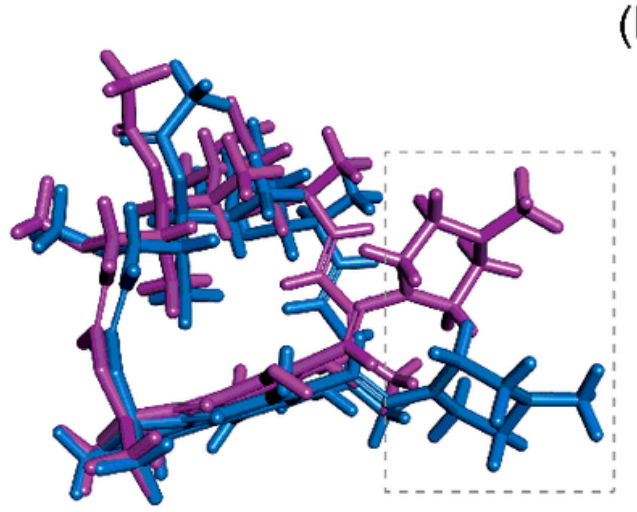

(D)

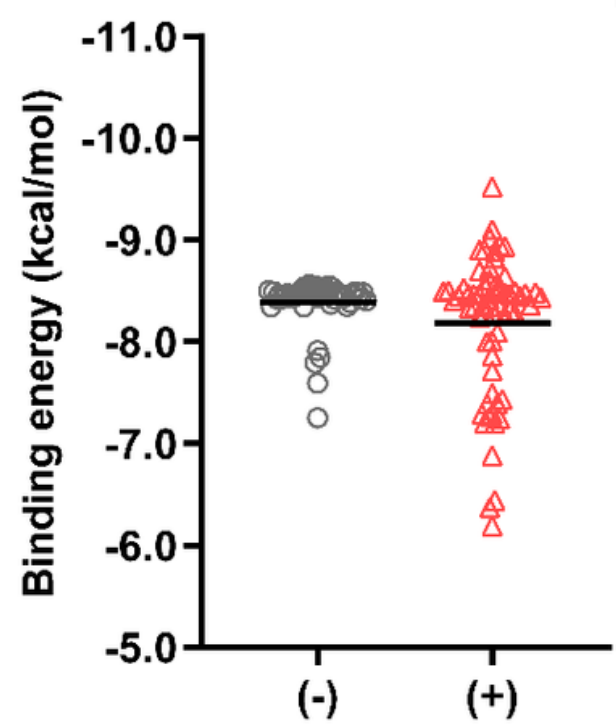

(B)

(C)
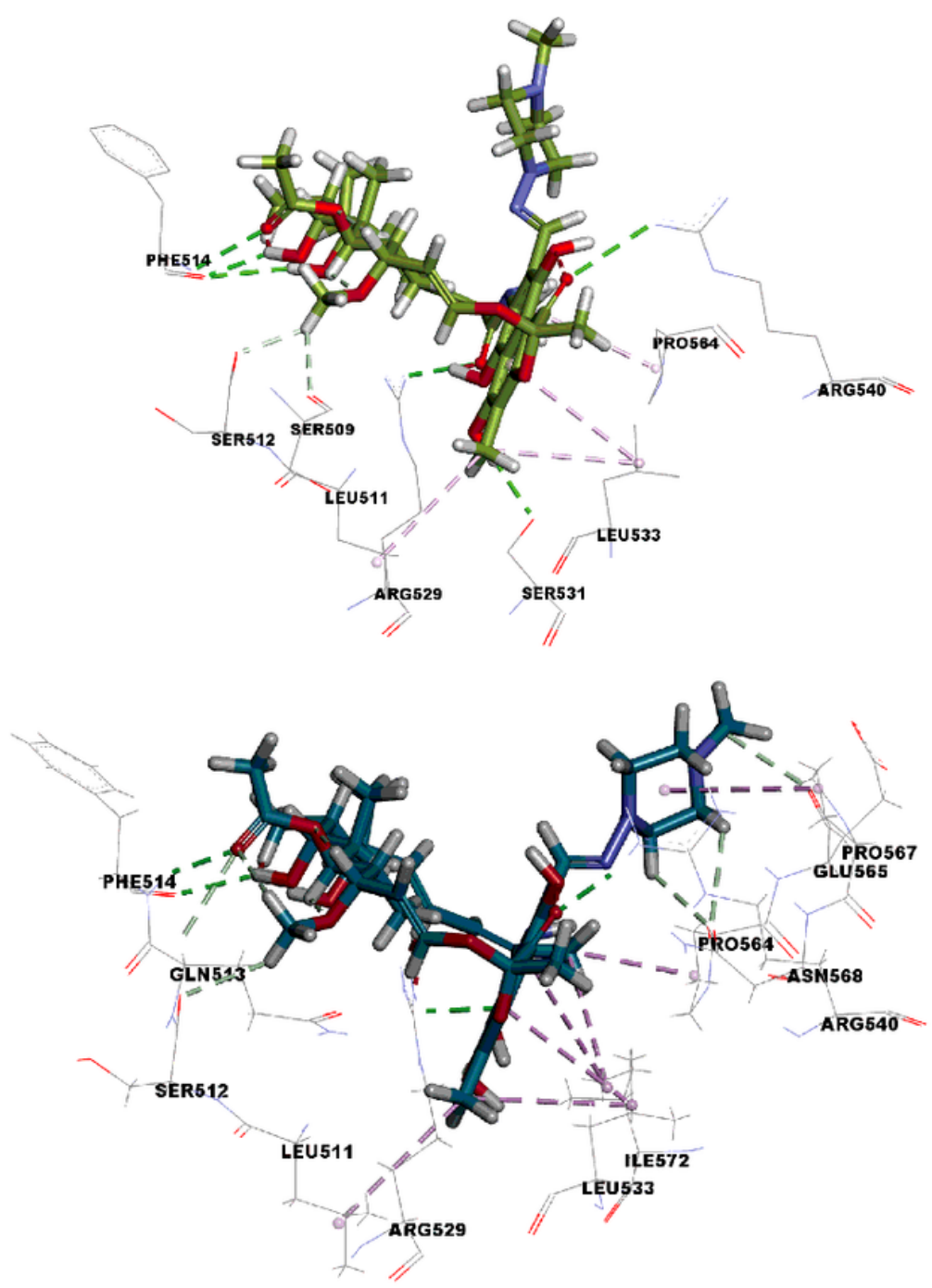

Mutation type

Figure 3

(A) Comparison of the Rifampin (Rif) poses obtained by molecular docking in the present research (purple) and obtained experimentally in the literature (blue). The structure in the dashed box is the 1-methylpiperazine tail of Rif. (B) Interactions between wild type RpoB and Rif molecule in the RpoB-Rif complex obtained from the Protein Database (5UAC). (C) Interactions between wild type RpoB and Rif molecule in RpoB-Rif complex obtained by molecular docking. (D) Distributions of the binding energies of Rif with RpoB mutants. The straight lines indicate the mean values. 
(A)
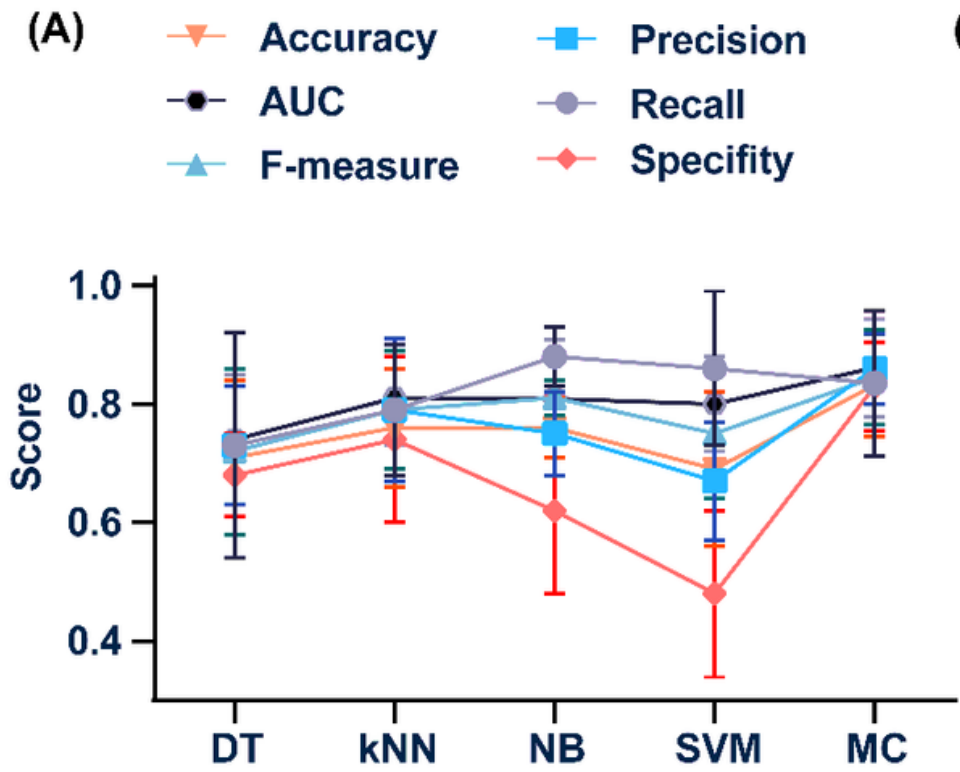

(B)

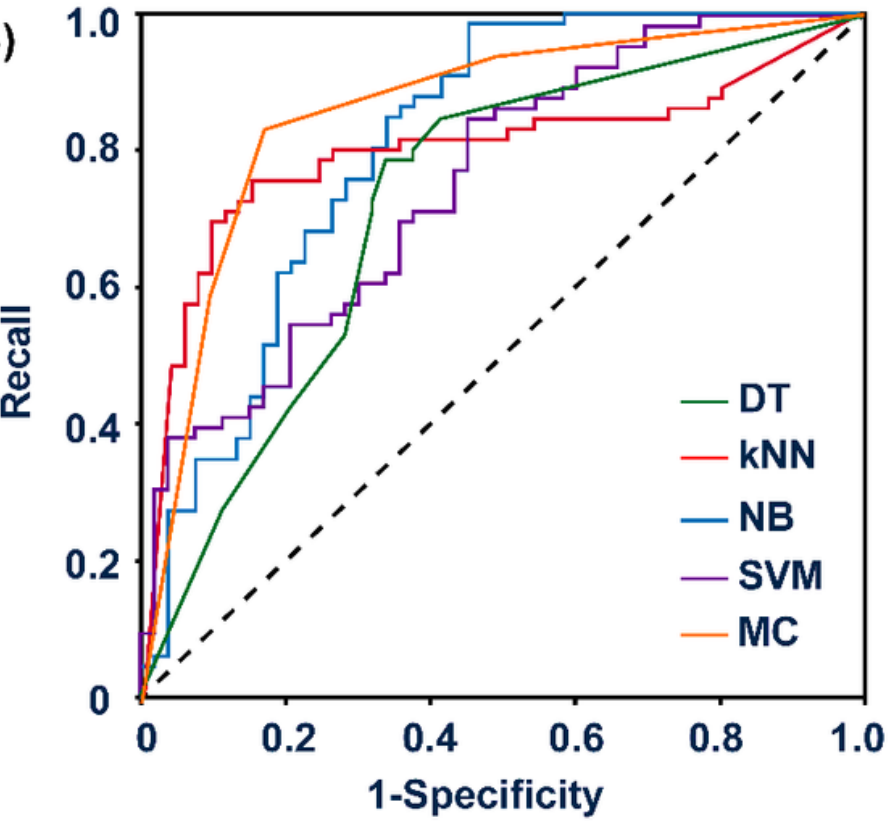

Figure 4

(A) Comparison of the prediction results of the developed classifiers. DT: decision tree; kNN: $k$ nearest neighbors; NB: naïve bayes; SVM: supporting vector machine. AUC: area under the curve. B) Receiver operating characteristic (ROC) curves for the four predictive models.

(A)
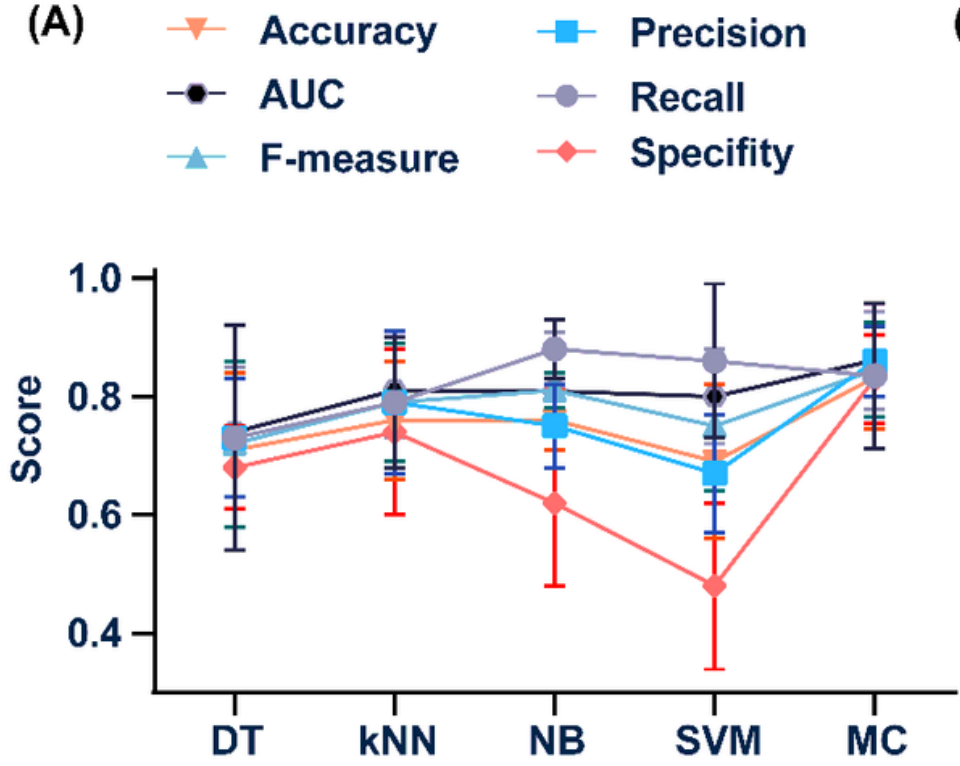

(B)

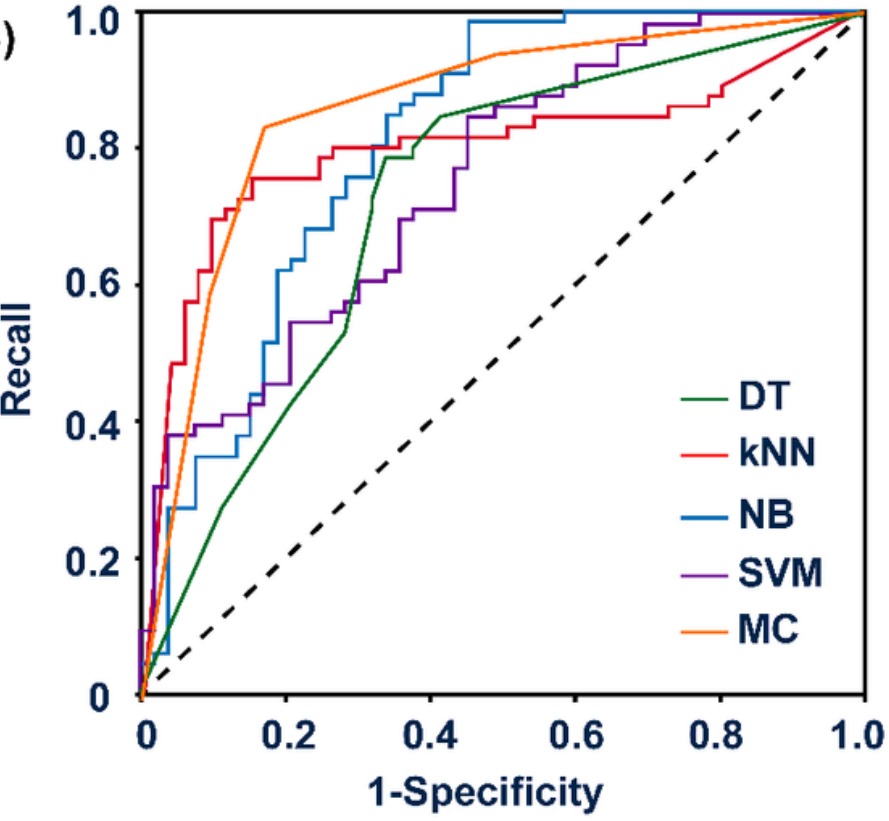

Figure 4

(A) Comparison of the prediction results of the developed classifiers. DT: decision tree; kNN: k nearest neighbors; NB: naïve bayes; SVM: supporting vector machine. AUC: area under the curve. B) Receiver operating characteristic (ROC) curves for the four predictive models. 


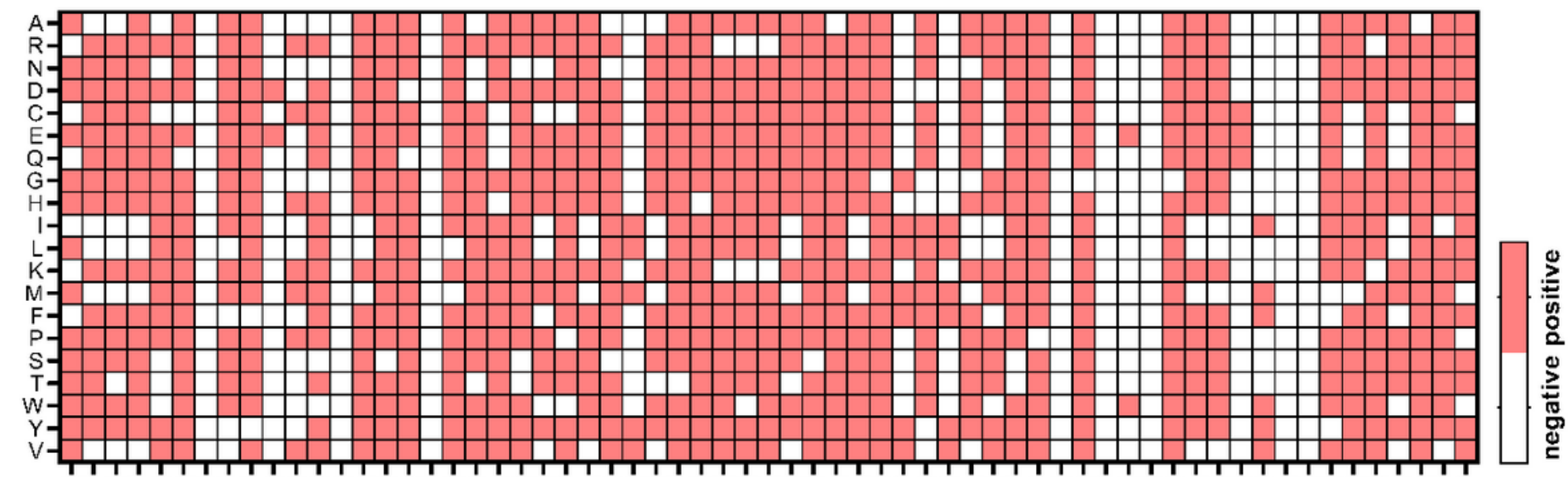

脊殀

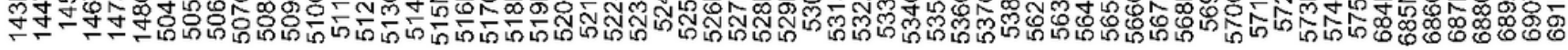

Figure 5

Resistance types (negative or positive) of the mutations occurring within RRDR regions in RpoB of E. coli. X axis labels represent the location and the single-letter abbreviation of the amino acid residues, $y$ axis labels are the abbreviation of the mutated residues.

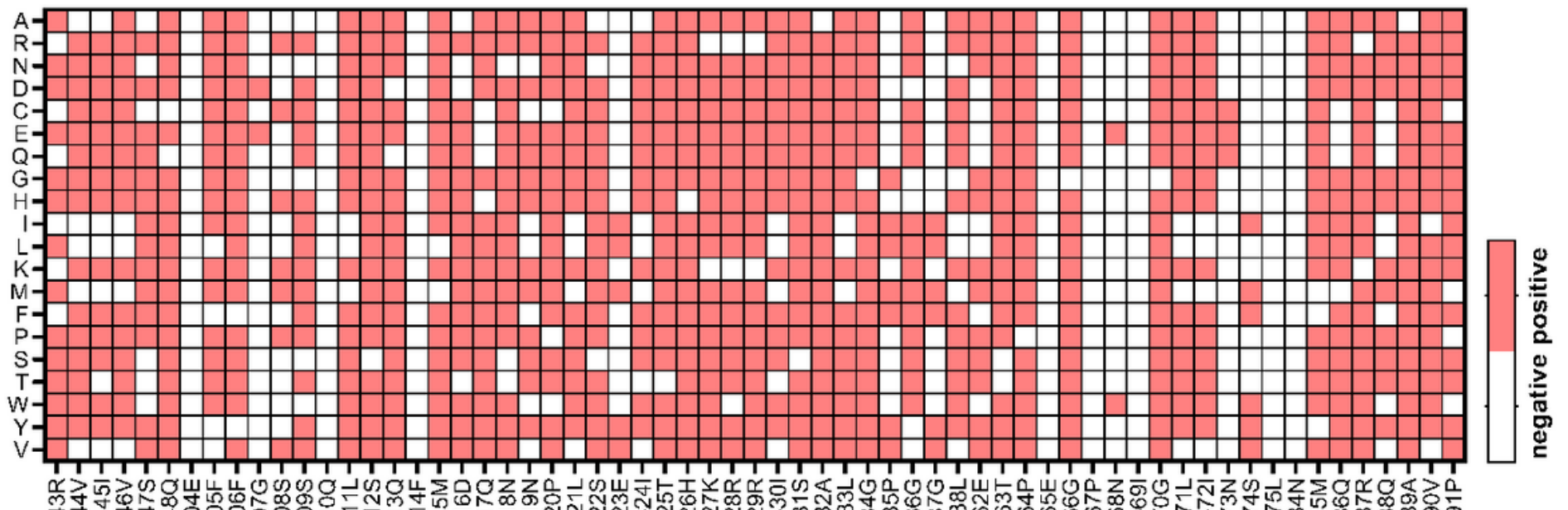

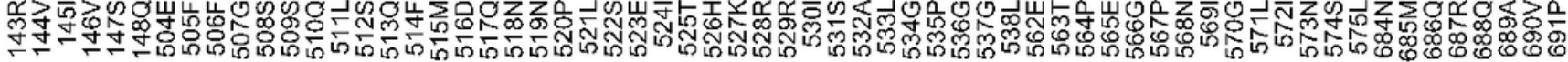

\section{Figure 5}

Resistance types (negative or positive) of the mutations occurring within RRDR regions in RpoB of E. coli. X axis labels represent the location and the single-letter abbreviation of the amino acid residues, $y$ axis labels are the abbreviation of the mutated residues.

\section{Supplementary Files}

This is a list of supplementary files associated with this preprint. Click to download.

- SupportingInformation.docx

- SupportingInformation.docx 